\title{
Tributos sobre a Folha ou sobre o Faturamento? Efeitos Quantitativos Para o Brasil $^{*}$
}

\author{
Tiago V. Cavalcanti ${ }^{\dagger}$
}

Sumário: 1. Introdução; 2. Modelo Teórico; 3. Resultados Quantitativos; Economia Informal; 5. Conclusão; A. Método Numérico.

Palavras-chave: Reforma tributária; Bem-estar; Produto per capita; Emprego.

Códigos JEL: E62; H21; 023.

Este artigo estuda os impactos alocativos e de bem-estar de uma reforma tributária que substitui os impostos sobre a folha de salários por outro que incide sobre a receita. Para isso, desenvolve-se um modelo neoclássico de crescimento econômico com impostos sobre a receita e sobre a folha de salários. As simulações com parâmetros da economia brasileira mostram que tal reforma tributária tem um efeito negativo no bem-estar, no estoque de capital de longo prazo e consequentemente no produto per capita de longo prazo. Contudo, o emprego aumenta.

This paper studies the impacts of a tax reform which substitutes taxes on labor income by taxes on total revenue. In order to accomplish that, we construct and solve a general equilibrium neoclassical growth model with taxes on labor income and total revenue. The numerical simulations with Brazilian parameters show that such tax reforms would negatively affect welfare, the capital stock, and consequently per capita output. However, employment would increase. Such effects are quantitatively important.

\section{INTRODUÇÃO}

(Auerbach, 1996) argumenta que a reforma tributária deve incentivar o investimento e a acumulação de capital, que com isso levaria a um aumento do produto e da produtividade da economia no longo prazo, com efeitos positivos sobre o bem-estar. (Slemrod, Joel, and Jon Bakija, 1996) enfatizam também a necessidade de tornar o sistema tributário claro e simples, para, com isso, diminuir a evasão fiscal

\footnotetext{
*Agradeço Alexandre Rands de Barros, o editor Ricardo Cavalcanti e um parecerista anônimo por comentários. Os erros remanescentes são de minha responsabilidade.

${ }^{\dagger}$ Faculty of Economics, University of Cambridge and PIMES/UFPE. Email: E-mail: tvdvc2@cam.ac .uk.
} 
e aumentar a base de arrecadação de impostos da economia. ${ }^{1}$ (Prescott, 2002) mostra como o sistema tributário pode ter efeitos negativos sobre o emprego e com isso sobre a renda per capita.

Uma importante discussão corresponde à incidência dos tributos. Deve o imposto incidir sobre a folha de salários ou sobre o faturamento das empresas? Essa questão levou à reforma do sistema tributário brasileiro, que substituiu parte das contribuições sociais sobre a folha de salários por outra que tem incidência sobre o faturamento. Esta reforma tinha como objetivo diminuir o custo da mão-deobra formal, e com isso estimular a formalização das relações de trabalho no Brasil.

Será que esta substituição da contribuição social é um instrumento eficiente de alcançar um dos principais objetivos da reforma, que é o aumento do estoque de capital e produtividade da economia? Este artigo investiga exatamente esta questão. Para isso, o artigo desenvolve e resolve numericamente um sistema econômico dinâmico com tributos sobre a receita e sobre o custo de mão-de-obra das firmas.

O arcabouço teórico desenvolvido é baseado no modelo tradicional de crescimento econômico com trabalho variável. Artigos recentes investigam questões similares às estudadas aqui, usando o mesmo instrumental, mas para outros países (ver (Lucas, 1990), (McGrattan e Prescott, 2005) e (Prescott, 2002)). De fato, (Chari e McGrattan, 2007) mostram que vários modelos econômicos, incluindo aqueles com diferentes distorções e micro-fundamentos, ${ }^{2}$ são, do ponto de vista macroeconômico, equivalentes ao modelo neoclássico de crescimento com distorções sobre o trabalho, acumulação de capital e produtividade. Usando esta idéia, (Prescott, 2002) mostra que o produto por trabalhador na França está 30\% mais baixo do que o produto por trabalhador americano. A razão desta depressão da França relativa aos Estados Unidos vem das distorções no mercado de trabalho (ex., regulação, leis e impostos) francês. Apesar da produtividade dos trabalhadores nos dois países serem semelhantes, o número de horas trabalhadas média de um trabalhador francês é bem menor do que o de um trabalhador americano.

Os parâmetros do modelo foram calibrados usando técnicas usuais (ver Cooley e Prescott (1995)) e estudos recentes sobre a economia brasileira (ver (Bugarin, M., Ellery Jr., R., Gomes, V., A. Teixeira, 2003) e (Gomes, V., M. Bugarin, and R. Ellery Jr., 2005)). Assume-se nas simulações que a substituição proposta é neutra em relação à receita do governo de longo prazo. Ou seja, a reforma não muda a receita fiscal de longo prazo. Os exercícios quantitativos sugerem que uma simples substituição da taxa da contribuição social de $25 \%$ sobre a folha por outra de $13.5 \%$ sobre a receita resultaria em: ${ }^{3}$

- Uma redução em $16.5 \%$ no estoque de capital por trabalhador (queda no investimento) de longo prazo;

- Uma queda de 4.5\% no produto por trabalhador de longo prazo;

- Um aumento de 4\% no nível do emprego de longo prazo, devido as menores distorções no mercado de trabalho;

- Um efeito negativo de Bem-Estar de longo prazo na ordem de $2.5 \%$ do consumo anual.

Além desta introdução este artigo tem mais quatro secções. A secção 1 descreve o modelo teórico. A seç̧ão 3 resolve o modelo numericamente e simula os resultados quantitativos. A secção 4 discute a relação entre sistema tributário e setor informal. A última secção contém as conclusões.

\footnotetext{
${ }^{1}$ Argumento baseado na curva de Laffer mostra que a receita do governo não necessariamente cresce com o aumento de impostos. Isto porque a base fiscal tem uma relação inversa com o nível dos impostos.

${ }^{2}$ Por exemplo, modelos com restrição ao crédito, ou com falhas no sistema judiciário.

${ }^{3} \mathrm{O}$ imposto de $13.5 \%$ sobre o faturamento gera a mesma receita fiscal de longo prazo de uma taxa de $25 \%$ sobre a folha de salários. Estudo do (da Fazenda do Brasil, 2003) mostrava que, para aqueles trabalhadores recebendo um salário mínimo, a diferença entre o que a firma pagava e o que o trabalhador recebia representava $25 \%$ do custo total do emprego.
} 


\section{MODELO TEÓRICO}

Esta secção desenvolve uma simples versão do modelo de crescimento econômico com impostos cobrados com base na no custo da mão-de-obra e no faturamento. Com esse simples arcabouço, nós temos os principais ingredientes para estudar os efeitos de uma mudança nos impostos, tanto no curto como no longo prazo.

Tempo é discreto e indeterminado nesta economia, $t=0,1,2, \ldots$ Em cada período, há três mercadorias que são comercializadas: um bem de consumo e os insumos trabalho e capital.

\subsection{Empresas}

Existe um número finito, $j=1, \ldots, J$ de empresas nesta economia. As empresas empregam trabalho, $n^{j}$, e capital, $k^{j d}$, para produzir o bem de consumo. A tecnologia de cada empresa é representada pela sua função de produção:

$$
y_{t}^{j}=F\left(A_{t} n_{t}^{j}, k_{t}^{j d}\right)
$$

$F(.,$.$) é contínua, diferenciável, crescente nos dois argumentos, côncava e exibe retornos constantes$ à escala. Ou seja, o produto duplica com a duplicação dos fatores de produção. $A_{t}$ corresponde ao fator de produtividade da força de trabalho e $A_{t+1}=(1+\gamma) A_{t}$, onde $\gamma$ é a taxa do crescimento tecnológico.

Seja $w_{t}$ e $r_{t}$ o preço do aluguel do trabalho e do capital em termos do bem de consumo no período $t$, respectivamente. $O$ objetivo de cada empresa $j$ é escolher as alocações de trabalho e capital $\left\{\mathbf{n}^{j}{ }_{t}, \mathbf{k}^{j d}{ }_{t}\right\}$ em cada período a fim de maximizar o valor presente de seu lucro. Mais especificamente,

$$
\pi=n^{j}, k^{j d} \operatorname{Max}\left\{\sum_{t=0}^{\infty} p_{t}\left[\left(1-\tau_{t}^{y}\right) F\left(A_{t} n_{t}^{j}, k_{t}^{j d}\right)-\left(1+\tau_{t}^{n}\right) w_{t} n_{t}^{j}-r_{t} k_{t}^{j d}\right] .\right.
$$

Onde $\mathrm{p}_{t}$ é o preço do bem de consumo no período $\mathrm{t}$ em termos do bem de consumo no período zero, $\tau^{n}$ corresponde à taxa do imposto que incide sobre a folha de salarial, e $\tau^{y}$ representa o imposto sobre a receita da empresa. As firmas tomam os preços e os impostos como dados para maximizar os lucros. As condições de primeira ordem associadas a este problema são:

$$
\begin{gathered}
r_{t}=\left(1-\tau_{t}^{y}\right) F_{k}\left(A_{t} n_{t}^{j}, k_{t}^{j d}\right), \\
w_{t}=\frac{\left(1-\tau_{t}^{y}\right)}{\left(1+\tau_{t}^{n}\right)} F_{n}\left(A_{t} n_{t}^{j}, k_{t}^{j d}\right) A_{t} .
\end{gathered}
$$

Ou seja, os fatores de produção são pagos de acordo com suas produtividades marginais. Os impostos distorcem a taxa de retorno dos dois fatores produtivos. Note, contudo, que, enquanto o imposto sobre a folha de salários afeta diretamente apenas o preço da mão-de-obra, o imposto sobre a receita afeta diretamente o preço dos dois fatores.

\subsubsection{Famílias}

A economia é habitada por $\mathrm{i}=1,2, \ldots ., \mathrm{I}$, famílias, que vivem por um período indeterminado. Cada família é composta de $N_{t}$ membros no período t, onde

$$
N_{t+1}=(1+\eta) N_{t}
$$

e $\eta$ corresponde à taxa de crescimento populacional. ${ }^{4} N_{0}$, é normalizado a 1 . Cada membro de uma família é dotado de uma unidade de tempo produtivo em cada período, que pode ser alocado entre trabalho e lazer. A função utilidade que representa cada família i é dada por:

${ }^{4} \mathrm{O}$ tamanho da família inicial, 


$$
U^{i}=\sum_{t=0}^{\infty} \beta^{t} N_{t} u\left(c_{t}^{i}, l_{t}^{i}\right)
$$

Onde $\mathrm{c}^{i}{ }_{t}$ e $\mathrm{l}^{i}{ }_{t}$ correspondem ao consumo e ao lazer de um membro da família i no período t, respectivamente, e $\beta \in(0,1)$ é o fator de desconto subjetivo das famílias. ${ }^{5}$ Se $\mathrm{h}^{i}{ }_{t}$ é a fração do tempo dedicado ao trabalho, nós temos que $\mathrm{h}_{t}^{i}+\mathrm{l}^{i}{ }_{t}=1$.

As famílias são proprietárias do capital, os quais são alugados às empresas. O estoque de capital inicial da economia é dado, $\mathrm{K}_{0}$, e cada unidade domiciliar têm uma fração idêntica deste capital. Capital pode ser acumulado ao longo do tempo, de acordo com a seguinte equação:

$$
k_{t+1}^{i}=(1-\delta) k_{t}^{i}+i_{t}^{i},
$$

onde $\delta \in(0,1)$ é a taxa de depreciação e $\mathrm{i}_{t}$ corresponde ao investimento no período t. O problema que descreve o objetivo de uma família representativa é escolher $\left\{\mathrm{c}_{t}^{i}, \mathrm{~h}^{i}{ }_{t}, \mathrm{k}^{i}{ }_{t+1}\right\}$ para maximizar a utilidade sujeito à

$$
\sum_{t=0}^{\infty} p_{t}\left[w_{t} N_{t} h_{t}^{i}+r_{t} k_{t}^{i}+(1-\delta) k_{t}^{i}+T_{t}^{i}-N_{t} c_{t}^{i}-k_{t+1}^{i}\right] \geq 0
$$

Dado $k_{0}^{i} \mathrm{e}$

$$
h_{t}^{i}+l_{t}^{i}=1 \text {. }
$$

$\mathrm{T}_{t}$ são transferências lump-sum do governo para as famílias. A restrição acima sugere que o valor presente do consumo e do investimento não pode ser maior do que o valor presente da renda das famílias. As condições de primeira ordem deste problema são:

$$
\begin{gathered}
\frac{u_{l}\left(c_{t}^{i}, 1-h_{t}^{i}\right)}{u_{c}\left(c_{t}^{i}, 1-h_{t}^{i}\right)}=w_{t}, \\
u_{c}\left(c_{t}^{i}, 1-h_{t}^{i}\right)=\beta u_{c}\left(c_{t+1}^{i}, 1-h_{t+1}^{i}\right)\left[r_{t+1}+(1-\delta)\right], \\
\lim _{T \rightarrow \infty} u_{c}\left(c_{T}^{i}, 1-h_{T}^{i}\right) k_{T+1}^{i}=0 .
\end{gathered}
$$

A primeira equação representa o trade-off entre consumo e lazer e a segunda equação o trade-off entre consumo presente e futuro. A última equação é a condição de transversalidade, que sugere que, em uma data terminal, ou a utilidade marginal do consumo é zero, ou o estoque de capital deve ser nulo.

Definição: Um equilíbrio competitivo para esta economia é um vetor de preços $\left\{\mathrm{p}_{t}, \mathrm{w}_{t}, \mathrm{r}_{t}\right\} \forall \mathrm{t}$, um vetor de políticas $\left\{\mathrm{T}_{t}, \tau^{n}{ }_{t}, \tau^{y}{ }_{t}\right\} \forall \mathrm{t}$, um vetor de alocações para cada família $\left\{\mathrm{c}^{i}{ }_{t}, \mathrm{k}^{i}{ }_{t+1}, \mathrm{~h}^{i}{ }_{t}\right\} \forall \mathrm{t}$, e um vetor de alocações para cada firma $\left\{\mathrm{n}^{j}{ }_{t}, \mathrm{k}^{j d}{ }_{t}\right\} \forall \mathrm{t}$, tal que

1. Dado os preços $\left\{\mathrm{p}_{t}, \mathrm{w}_{t}, \mathrm{r}_{t}\right\}$ e políticas $\left\{\mathrm{T}_{t}, \tau^{n}{ }_{t}, \tau^{y}{ }_{t}\right\} \forall \mathrm{t},\left\{\mathrm{c}^{i}{ }_{t}, \mathrm{k}^{i}{ }_{t+1}, \mathrm{~h}^{i}{ }_{t}\right\}$ resolve o problema de cada família $\mathrm{i}=1,2, \ldots, \mathrm{I}$.

2. Dado os preços $\left\{\mathrm{p}_{t}, \mathrm{w}_{t}, \mathrm{r}_{t}\right\}$ e políticas $\left\{\mathrm{T}_{t}, \tau^{n}{ }_{t}, \tau^{y}{ }_{t}\right\} \forall \mathrm{t},\left\{\mathrm{n}^{j}{ }_{t}, \mathrm{k}^{j d}{ }_{t}\right\}$ resolve o problema de cada firma $\mathrm{j}=1,2, \ldots, \mathrm{J}$.

\footnotetext{
${ }^{5}$ Assim como a função de produção, nós assumimos que a função utilidade em cada período, u(c, l), é contínua, diferenciável, crescente nos dois argumentos, côncava e satisfaz as condições de INADA.
} 
3. Equilíbrio de mercado:

Mercado de insumos:

$$
\begin{gathered}
L_{t}=\sum_{j=1}^{J} n_{t}^{j}=\sum_{i=1}^{I} N_{t} h_{t}^{i} ; \\
K_{t}=\sum_{j=1}^{J} k_{t}^{j d}=\sum_{i=1}^{I} k_{t}^{i} .
\end{gathered}
$$

Mercado de bens de consumo:

$$
C_{t}+K_{t+1}=F\left(K_{t}, A_{t} L_{t}\right)+(1-\delta) K_{t},
$$

onde

$$
C_{t}=\sum_{i=1}^{I} N_{t} c_{t}^{i}
$$

4. Governo tem um orçamento equilibrado:

$$
G_{t}=\sum_{i=1}^{I} T_{t}^{i}=\tau_{t}^{y} Y_{t}+\tau_{t}^{n} w_{t} L_{t}
$$

Condições (1) e (2) sugerem que as firmas e as famílias maximizam seus objetivos dados às restrições orçamentária e tecnológica. Condição 3 implica que a oferta e a demanda dos insumos e do bem de consumo são iguais. Condição 4 mostra que o orçamento do governo está equilibrado em cada período. Note que não há heterogeneidade entre as famílias, portanto, pode-se trabalhar com uma família representativa. $O$ mesmo se aplica para as firmas.

Usando as condições de primeira ordem das firmas e das famílias e as condições de equilíbrio de mercado, mostra-se que o sistema que descreve esta economia é dado por: ${ }^{6}$

$$
\begin{gathered}
\frac{u_{l}\left(C_{t}, 1-h_{t}\right)}{u_{c}\left(C_{t}, 1-h_{t}\right)}=\frac{\left(1-\tau_{t}^{y}\right)}{\left(1+\tau_{t}^{n}\right)} F_{n}\left(A_{t} N_{t} h_{t}, K_{t}\right), \\
u_{c}\left(C_{t}, 1-h_{t}\right)=\beta u_{c}\left(C_{t+1}, 1-h_{t+1}\right)\left[\left(1-\tau_{t+1}^{y}\right) F_{k}\left(A_{t+1} N_{t+1} h_{t+1}, K_{t+1}\right)+(1-\delta)\right], \\
C_{t}+K_{t+1}=F\left(A_{t} N_{t} h_{t}, K_{t}\right)+(1-\delta) K_{t}, \\
\lim _{T \rightarrow \infty} \beta^{T} u_{c}\left(C_{T}, 1-h_{T}\right) K_{T+1}=0, \\
A_{t+1}=(1+\gamma) A_{t}, \\
N_{t+1}=(1+\eta) N_{t} .
\end{gathered}
$$

O estoque inicial do capital $K_{0}$ é dado. Note que o imposto que incide sobre a folha de salários afeta diretamente a escolha marginal entre consumo e lazer, mas não afeta diretamente a condição marginal

\footnotetext{
${ }^{6}$ As variáveis maiúsculas correspondem as variáveis agregadas.
} 
entre consumo hoje e consumo futuro (poupança, ou acumulação de capital). Note, entretanto, que existe um efeito indireto, via seu impacto sobre o nível de emprego e consumo. 0 imposto que incide sobre a receita, contudo, tem um efeito direto nas duas condições marginais de equilíbrio.

\section{RESULTADOS QUANTITATIVOS}

A fim de resolver o modelo numericamente é necessário definir formas funcionais para a tecnologia e preferências, como também determinar os valores dos parâmetros do modelo. A função de produção utilizada é a Cobb-Douglas

$$
F\left(K_{t}, A_{t} N_{t} h_{t}\right)=K_{t}^{\theta}\left(\pi A_{t} N_{t} h_{t}\right)^{1-\theta},
$$

onde $\theta \in(0,1)$ é a fração do produto proveniente do capital e $\pi$ corresponde a distância da tecnologia adotada em relação à fronteira tecnológica $A_{t}$. A função utilidade é dada por u(c, l) $=\ln (\mathrm{c})+\alpha \ln (\mathrm{l})$, onde $\alpha>0$ é o peso do lazer em relação ao consumo na utilidade das famílias. Tais formas paramétricas da função utilidade e da função de produção, são comuns em macroeconomia. ${ }^{7}$ 3.1) Determinação dos Parâmetros

Os parâmetros estruturais foram determinados com base em técnicas tradicionais (ver Cooley e Prescott (1995)) de calibração, utilizando também estudos recentes da economia brasileira (ver (Bugarin, M., Ellery Jr., R., Gomes, V., A. Teixeira, 2003), e (Gomes, V., M. Bugarin, and R. Ellery Jr., 2005)). Cada período no modelo é interpretado como sendo um ano. Assume-se que a economia está no seu equilíbrio de longo prazo, onde: a política do governo é estacionária,

$$
\tau_{t+1}^{l}=\tau_{t}^{j},
$$

para $\mathrm{j}=\mathrm{n}$, y; o estoque de capital, o produto, e o consumo agregado crescem a uma mesma taxa, dada por $(1+\gamma)(1+\eta)$. Neste equilíbrio, em particular, a condição marginal entre consumo e lazer é dada por $^{8}$

$$
\begin{gathered}
\hat{Y}_{t}=\frac{Y_{t}}{A_{t} N_{t}}, \\
\hat{C}_{t}=\frac{C_{t}}{A_{t} N_{t}}, \\
\hat{I}_{t}=\frac{I_{t}}{A_{t} N_{t}}, \\
\hat{K}_{t}=\frac{K_{t}}{A_{t} N_{t}},
\end{gathered}
$$

correspondem ao produto, consumo, investimento e ao estoque de capital agregado de longo prazo por unidade de eficiência da força de trabalho. Tal transformação é necessária para tornar a economia em um sistema estacionário.

$$
\frac{\alpha h}{1-h}=\frac{\left(1-\tau^{y}\right)}{\left(1+\tau^{n}\right)} \frac{\hat{Y}}{\hat{C}} .
$$

A condição marginal entre consumo presente e futuro é

\footnotetext{
${ }^{7}$ Ver, por exemplo, Cooley e Prescott (1995) e (Prescott, 2002).

${ }^{8}$ As variáveis
} 


$$
\frac{\hat{Y}}{\hat{K}}=\frac{1}{1-\tau^{y}}\left[\frac{1+\gamma}{\beta}-(1-\delta)\right] .
$$

A equação que ilustra a evolução do capital é:

$$
\delta=\frac{\hat{I}}{\hat{K}}+1-(1+\gamma)(1+\eta) .
$$

Como a questão a ser investigada é a substituição de alguns impostos que incidem sobre a folha de salários por um que incida sobre o faturamento, nós vamos abstrair de todos os outros impostos da economia e considerar apenas estes que incidem sobre a folha de salários. Isto implica que inicialmente $\tau^{y}=0$, como também os impostos sobre o consumo, e sobre a renda proveniente do capital, que não foram explicitamente definidos no modelo.

A simulação da reforma tributária irá se basear no documento do ministério da fazenda de 2003 sobre política econômica e reforma estrutural. De acordo com este estudo, para um trabalhador que recebe um salário mínimo, a diferença entre o que a firma paga e o que o trabalhador recebe representa $25 \%$ do custo total do emprego. No modelo, isto implica que $\tau^{n}=0.25$.

Estimativas de (Bugarin, M., Ellery Jr., R., Gomes, V., A. Teixeira, 2003) sugerem que a participação do capital na produção brasileira é de $35 \%$, ou seja, $\theta=0.35^{9}$. A razão consumo-produto teve uma média de 0.65 entre 1980 e $1998^{10}$. Usando a equação do trade-off entre consumo e lazer no estado estacionário, os valores de $\theta, \mathrm{Y} / \mathrm{C}$,

$$
\tau^{n}, \tau^{y}
$$

e o fato estilizado de que os trabalhadores, em geral, gastam um terço do tempo disponível no mercado (ver (Hansen e Wright, 1996)), nós temos que o peso do lazer na função utilidade é de 1.6, ou seja $\alpha=1.6$.

De acordo com (Gomes, V., M. Bugarin, and R. Ellery Jr., 2005), a taxa anual de crescimento populacional para o Brasil entre 1947 e 1998 foi de 2\%, o que implica que $\eta=0.02$. (Parente e Prescott, 2000) sugerem que

$$
(1+\gamma)^{1-\theta}
$$

corresponde ao crescimento da fronteira tecnológica do progresso técnico no mundo ${ }^{11}$ e que para o último século a taxa anual de crescimento tecnológico foi em torno de $2 \%$. Ou seja

$$
(1+\gamma)^{1-\theta}=1.02 .
$$

Como $\theta=0.35$, nós temos que $\gamma=0.013$. Para o período entre 1980-metricconverterProductID1998, a1998, a razão capital-produto, $\mathrm{K} / \mathrm{Y}$, e investimento-produto, teve uma média de 2.5 e 0.35 , respectivamente. ${ }^{12} \mathrm{O}$ que implica em uma razão investimento-capital de 0.14 . Usando a equação de acumulação de capital no longo prazo, nós temos uma taxa de depreciação de $10 \%$.

Como o produto por trabalhador no Brasil ficou em torno de $30 \%$ do produto por trabalhador nos Estados Unidos nos últimos 30 anos, isto implica que $\pi=0.30$ (Heston and Summers (2002)). Usando a taxa de depreciação, a razão capital-produto, e a equação que determina o trade-off entre consumo presente e futuro no longo prazo, nós temos que o fator subjetivo de desconto é $\beta=0.97$.

\footnotetext{
${ }^{9}$ As estimativas de (Bugarin, M., Ellery Jr., R., Gomes, V., A. Teixeira, 2003) da participação do capital na renda total são consistentes com as estimativas de (Gollin, 2002) para vários países.

${ }^{10}$ Ver tabela 2 de (Bugarin, M., Ellery Jr., R., Gomes, V., A. Teixeira, 2003). Aqui nós estamos abstraindo dos gastos do governo.

${ }^{11}$ Países mais pobres podem sempre adotar as tecnologia geradas nos países mais ricos.

${ }^{12}$ Ver tabelas 2 e 3 de (Bugarin, M., Ellery Jr., R., Gomes, V., A. Teixeira, 2003).
} 
A tabela 1 descreve e explica os valores dos parâmetros. Com os parefeitos no produto, acumulação do capital e emprego dos instrumentos de política do governo. Mais especificamente, $\tau^{n}{ }_{t}$ e $\tau^{y}{ }_{t}$

Tabela 1 - Valores dos Parâmetros

\begin{tabular}{ccc}
\hline$\beta$ & 0,97 & Fator de desconto \\
$\alpha$ & 1,6 & Peso relativo do lazer \\
$\eta$ & 0,02 & Taxa de crescimento populacional \\
$\theta$ & 0,35 & Participação relativa do capital na produção \\
$\gamma$ & 0,013 & Progresso técnico \\
$\pi$ & 0,3 & Distância relativa à fronteira tecnológica \\
$\delta$ & 0,1 & Taxa de depreciação do capital \\
$\tau^{n}$ & 0,25 & Taxa do imposto sobre a folha de salários \\
$\tau^{y}$ & 0 & Taxa do imposto sobre o faturamento \\
\hline
\end{tabular}

\subsection{Efeitos Quantitativos da Reforma}

Esta seção investiga os efeitos quantitativos da substituição do imposto sobre a folha de salários, $\tau^{n}$, por outro que incide sobre a receita, $\tau^{y}$, tal que a receita tributária de longo prazo permaneça constante. Ou seja, a reforma proposta é neutra em relação à receita do governo de longo prazo.

Figura 1 mostra os efeitos quantitativos da reforma sobre o produto por trabalhador (painel (a)); o estoque de capital por trabalhador (painel (b)); o número de horas trabalhadas de cada trabalhador (painel (c)); e sobre a receita tributária do governo (painel (d)). A figura mostra a dinâmica de transição de um equilíbrio de longo prazo, onde a taxa do imposto que incide sobre a folha de salários é de $25 \%$ e a taxa do imposto sobre a receita é zero, para outro equilíbrio em que o governo taxa apenas o faturamento e mantém o mesmo nível de receita inicial. Neste caso, $\tau^{n}=0$ e $\tau^{y}=0.135$.

Pode-se notar que o produto e o estoque de capital por trabalhador caem monotonicamente de um equilíbrio para o outro com a reforma. Contudo, o efeito negativo sobre o capital é mais forte. Enquanto o produto por trabalhador cai em $4 \%$, o efeito negativo sobre o capital é de $16 \%$. O menor efeito sobre o produto é, em parte, explicado pelo aumento de $4.5 \%$ no número de horas de trabalho. Note que com a diminuição do estoque de capital, a produtividade marginal por trabalhador diminui, reduzindo, assim, a demanda por horas trabalhadas. Contudo, a substituição do imposto sobre a folha de salários por outro que incide sobre a receita diminui as distorções no mercado de trabalho, levando a um aumento na demanda por este fator. $O$ efeito líquido positivo sobre o número de horas trabalhadas implica que, o efeito negativo da queda na produtividade é menor do que o efeito positivo da redução nas distorções no mercado de trabalho.

A receita do governo inicialmente cresce com a reforma. A razão é simples: Logo após a reforma o produto ainda está próximo do seu valor pré-reforma. Como o novo imposto incide sobre a receita e o produto de longo prazo do equilíbrio inicial é maior do que o produto de longo prazo pós-reforma, a receita do governo aumenta. À medida que o produto cai, a receita do governo diminui, até o seu valor inicial.

Quais os efeitos da reforma sobre o bem-estar dos agentes? Para isso, nós vamos usar o conceito de variação compensada, ${ }^{13}$ que mensura a percentagem da variação no consumo das famílias necessária para deixar as famílias no mesmo nível de bem-estar do período pré-reforma. Ou seja,

\footnotetext{
${ }^{13}$ Robert Lucas Jr. usa a mesma medida para estudar várias questões de natureza quantitativa para a economia americana. Por exemplo, (Lucas, 1990), através desta medida, calcula os efeitos de bem-estar de uma redução no imposto sobre a renda do capital. (Lucas, 2000) estuda os efeitos de bem-estar de uma redução da inflação de $10 \%$ para $0 \%$.
} 


$$
u\left((1+\omega) c\left(z^{\prime}\right), l\left(z^{\prime}\right)\right)-u(c(z), l(z))=0,
$$

onde $\mathrm{z}$ corresponde ao vetor de políticas antes da reforma,

$$
z=\left(\tau^{n}=0.25, \tau^{y}=0\right)
$$

z' o vetor pós-reforma,

$$
\left.z^{\prime n}=0, \tau^{y}=0.135\right),
$$

e $\omega$ é a variação percentual do consumo que deixaria as famílias no mesmo nível de bem-estar antes da reforma. Note que o cálculo corresponde apenas aos efeitos de bem-estar de longo-prazo, já que a equação acima abstrai do período de transição de um equilíbrio para o outro. ${ }^{14}$ Os resultados sugerem que a substituição da contribuição social sobre a folha de salários por outra que incida sobre o faturamento geraria um custo de bem-estar de longo-prazo de $2.48 \%$ do consumo anual das famílias. Ou seja, os agentes desta economia estariam dispostos a diminuir o consumo anual em até $2.48 \%$ para evitar a substituiçã o proposta pelo governo.

Figura 1 - Efeitos quantitativos do reforma
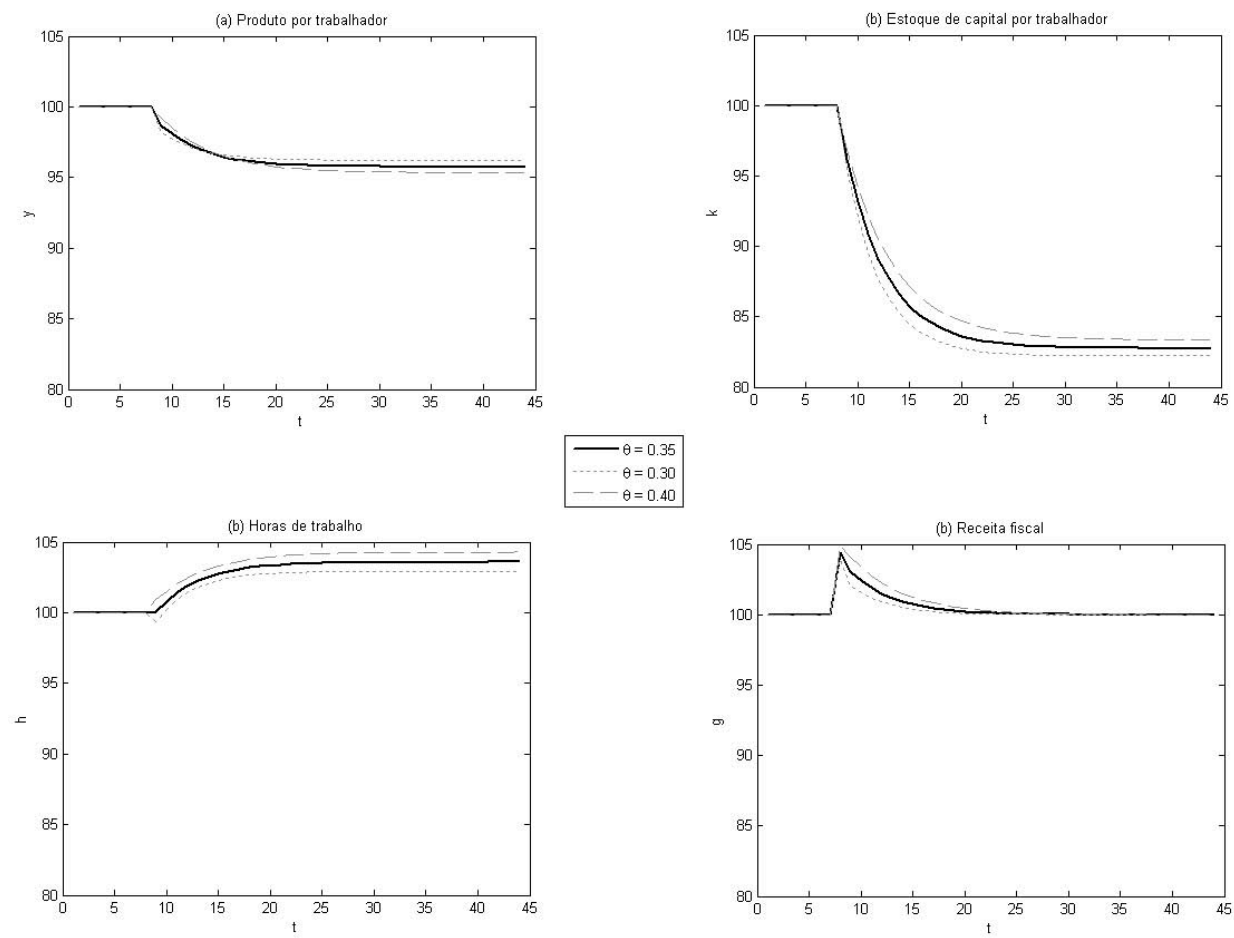

\footnotetext{
${ }^{14}$ Por não se considerar o período de transição, esse custo de bem-estar deve ser visto como um limite superior do verdadeiro custo de bem-estar da reforma. A razão é que a receita do governo eleva-se no período de transição e depois converge ao seu nível anterior. Porém como este adicional de receita é transferido às famílias, isto provoca um efeito sobre o bem-estar positivo no curto prazo. Comparações dos valores das utilidades presentes das duas políticas, ainda sugerem que o efeito de bem-estar é negativo.
} 


\subsection{Sensibilidade em Relação à Intensidade do Capital}

Os resultados acima foram gerados através de mudanças apenas nos instrumentos de política do governo. Contudo, os resultados podem ser diferentes dependendo da intensidade do capital na produção. Parâmetro $\theta$ mensura o grau de intensidade do capital na produção. Quanto maior for $\theta$, maior será a participação do capital na produção. No caso limite, quando $\theta$ é um, apenas o capital é importante na produção. Figura 1 mostra também a sensibilidade dos resultados em relação à participação relativa do capital na produção. Os efeitos sobre o produto, por exemplo, é maior quanto maior for à intensidade do capital na produção. Contudo, note que a variação nos resultados é quantitativamente insignificante. Portanto, os resultados são robustos à intensidade relativa do capital, $\theta$. Variações de $15 \%$ na participação relativa do capital na produção implicam em variações na ordem de menos de $1 \%$ nos resultados do modelo. A medida de bem-estar também varia pouco com a participação relativa do capital na renda total. ${ }^{15} \theta=0.40$ e $\theta=0.3$, respectivamente.

\subsection{Entendendo os Resultados Quantitativos}

Por que a substituição da contribuição social que incide sobre a folha de salários por outra que incide sobre o faturamento tem um efeito negativo sobre a produtividade e bem-estar? Note que, enquanto o imposto sobre o custo da mão-de-obra afeta diretamente apenas o trade-off entre consumo e lazer, o imposto sobre o faturamento afeta diretamente não só este trade-off, mas também a decisão de investimento (consumo presente e futuro). Ou seja, o imposto sobre a receita não só gera uma distorção no mercado de trabalho, como também na alocação do capital. Neste caso, o imposto sobre o faturamento é similar a um imposto sobre os rendimentos do capital. Um resultado conhecido na literatura sobre tributação ótima é o teorema de Chamley, que mostra que o imposto sobre os rendimentos do capital deve ser zero no longo prazo.

Este teorema segue um princípio básico de finanças públicas, que sugere que bens similares devem ser tributados a uma mesma taxa - uniform commodity taxation - (Atkinson, A. B., and J. E. Stiglitz, 1972). Como o imposto sobre os rendimentos do capital é similar a um imposto sobre a poupança, este imposto tributa o consumo futuro a uma taxa mais alta do que o consumo presente. Portanto, de acordo com princípio de (Atkinson, A. B., and J. E. Stiglitz, 1972), o imposto sobre os rendimentos do capital deve ser nulo no longo prazo. Embora, o teorema de Chamley tenha sido demonstrado em uma economia similar a estudada aqui, (Atkenson, A., V. V. Chari, and P. Kehoe, 1999) mostram que este resultado é também satisfeito em modelos mais complexos, com agentes heterogêneos ao invés de idênticos, crescimento endógeno, economia aberta e também em modelos de gerações sobrepostas. (Chari, 2000) mostram também que, dependendo do tipo da função utilidade, o imposto sobre o rendimento do capital deve ser zero não apenas no longo prazo, mas também depois do primeiro período. Com base neste princípio de finanças públicas, (Lucas, 1990, 293) conclui que "neither capital gains nor any of the income from capital should be taxed at all." Os resultados quantitativos seguem exatamente este princípio.

\section{SISTEMA TRIBUTÁRIO E A ECONOMIA INFORMAL}

Um dos objetivos da substituição proposta é estimular o aumento das relações formais de trabalho. ${ }^{16}$ Para entender se tal reforma teria um efeito positivo sobre o grau de formalização do trabalho é necessário entender os determinantes da economia informal. Um ponto que deve ser enfatizado é

\footnotetext{
${ }^{15} \mathrm{O}$ custo de bem-estar da reforma é de $2.49 \%$ e $2.47 \%$ quando

${ }^{16}$ Do ponto de vista do desenvolvimento econômico e social, o combate à evasão fiscal é fundamental já que aumenta os recursos para investimentos em setores estratégicos, como educação e programas de combate à pobreza. Do ponto de vista fiscal também, já que gera um aumento da receita do governo sem o aumento dos impostos.
} 
Tributos sobre a Folha ou sobre o Faturamento? Efeitos Quantitativos Para o Brasil

que as empresas não só empregam trabalhadores informalmente, mas também fazem qualquer tipo de contratos de compra e vende de produto e insumos sem cumprir as regras legais e fora da fiscalização da autoridade tributária.

Trabalhados empíricos recentes investigam exatamente os determinantes da economia informal. Primeiramente, os dados mostram que a participação do setor informal no produto total dos países é importante não só para os países em desenvolvimento, mas também para alguns países industrializados, como Espanha, Itália e Portugal (Schneider e Enste, 2000). Utilizando dados para países industrializados e PersonNameProductIDem desenvolvimento Friedman em desenvolvimento (Friedman, E., Johnson, S., Kaufmann, D., and P. Zoido-Lobaton, 2000) mostram que os principais determinantes do setor informal são: i) elevadas barreiras à formalização, na forma de custos legais, tanto em unidade monetária como em tempo; ii) corrupção; iii) fraco sistema de fiscalização e complexidade do sistema tributário e jurídico. Quanto ao impacto dos impostos sobre o tamanho do setor informal, os resultados não são claros. Por um lado, a maior carga tributária eleva o custo de permanecer legal, incentivando, assim, o setor informal. Mas, por outro, aumenta a receita do governo, que pode ser utilizada para o cumprimento e fiscalização das regrais legais. Neste caso, o ponto fundamental é a combinação da eficiência do sistema legal e a carga tributária.

Estudos teóricos corroboram tais resultados. (Dessy e Pallage, 2003) e (Antunes e Cavalcanti, 2007) desenvolvem modelos em que os agentes podem abrir um negócio (formal/informal) ou trabalhar como empregado em um dos dois setores. Para operar no setor informal as firmas pagam custos de legalidade e impostos. No setor informal, com certa probabilidade, as firmas não pagam tais custos e impostos, mas tem menor acesso ao crédito para financiar projetos e pagar o custo do capital. ${ }^{17}$ Exercícios quantitativos de simulação mostram que a eficiência do sistema legal e as fortes barreiras á formalização explicam grande parte da variação do tamanho do setor informal entre os países (ver (Antunes e Cavalcanti, 2007)). Enquanto os impostos e a incidência desses têm um efeito pequeno sobre o setor informal.

\section{CONCLUSÃO}

Este trabalho investigou os impactos econômicos da substituição da contribuição social que incide sobre a folha de salários por outra que incide sobre o faturamento. Para isso, o trabalho desenvolveu e resolveu numericamente um modelo de crescimento econômico neoclássico tradicional com tributos sobre a receita e sobre o custo da mão-de-obra. As formas funcionais e os parâmetros, que descrevem a tecnologia das firmas e a utilidade das famílias, foram determinados com base em métodos tradicionais de calibração e em estudo recente da economia brasileira. Os exercícios quantitativos sugerem que uma simples substituição da taxa da contribuição social de $25 \%$ sobre a folha por outra de $13.5 \%$ sobre a receita tem efeitos negativos substanciais sobre a acumulação de capital (redução de $16.5 \%$ ). Consequentemente, tal reforma tem efeitos negativos também sobre o produto de longo prazo (queda de 4.5\%). O emprego, por outro lado, aumenta em 4\%. Portanto, a reforma proposta aumenta o emprego, mas diminui a produtividade por trabalhador de longo prazo. Em termos de bem-estar a reforma gera um custo de longo prazo de aproximadamente $2.5 \%$ do consumo anual.

Com efeito, o artigo mostra que tal reforma não se justifica do ponto de vista econômico. A reforma tributária deve incentivar o investimento e a acumulação de capital, que com isso levaria a um aumento do produto e da produtividade da economia no longo prazo (ver (Auerbach, 1996)). A substituição da contribuição proposta teria um efeito inverso ao sugerido por argumentos baseados na eficiência do sistema econômico. Por fim, como sugerem (Slemrod, Joel, and Jon Bakija, 1996), a reforma tributária deve criar um sistema de imposto simples e claro, para diminuir a evasão fiscal e aumentar com isso

\footnotetext{
${ }^{17}$ Esta é a idéia de (De Soto, 2000), que coloca como uma das causas fundamentais do subdesenvolvimento dos países, a incapacidade dos agentes nesses países em gerar capital do próprio capital. Isto porque grande parte da riqueza nesses países não tem o status legal e portanto não pode ser usada em um empréstimo como colateral.
} 
a base de arrecadação da economia. Isto diminuiria a participação do setor informal na economia e a carga de impostos sobre os trabalhadores e firmas formais. A simples substituição da incidência da contribuição social também não se justifica sobre essa ótica, pois não simplifica o sistema de tributário. Se o objetivo é incentivar a formalização das relações de trabalho, o governo deve: 1) diminuir as fortes barreiras à legalidade (regulação, custos legais à entrada, corrupção); 2) melhorar o funcionamento do sistema de ordem e lei; 3) simplificar o sistema tributário; e 4) diminuir a carga dos tributos. A substituição proposta diminui a eficiência da economia e aumenta a carga tributária sem simplificar a cobrança dos impostos.

\section{Referências Bibliográficas}

Antunes, A. \& Cavalcanti, T. (2007). Start up costs, limited enforcement and the hidden economy. European Economic Review, 57(1):203-224.

Atkenson, A., V. V. Chari, and P. Kehoe (1999). Taxing capital income: A bad idea. Federal Reserve Bank of placeCityMinneapolis Quarterly Review, 23:3-17.

Atkinson, A. B., and J. E. Stiglitz (1972). The structure of indirect taxation and economic efficiency. Journal of Public Economics, 1:97-119.

Auerbach, A. J. (1996). Tax reform, capital allocation, efficiency, and growth. Economic Effects of Fundamental Tax Reform.

Bugarin, M., Ellery Jr., R., Gomes, V., A. Teixeira (2003). The brazilian depression in the 80s and 90s. The Depression Experiences of the $20^{\text {th }}$ Century, Federal Reserve Bank of Minneapolis.

Chari, V. V., P. K. \& McGrattan, E. (2007). Business cycle accounting. Econometrica, 75(3):781-836.

Chari, V.V., P. K. (2000). Opitmal fiscal and monetary policy. Handbook of Macroeconomics, 1.

da Fazenda do Brasil, M. (2003). Política econômica e reformas estruturais. Artigo disponível na página do Ministério da Fazenda (www.fazenda.gov.br).

De Soto, H. (2000). The mystery of capital. Harper and Row.

Dessy, S. \& Pallage, S. (2003). Taxes, inequality, and the size of the informal sector. Journal of Development Economics, 70(1):225-233.

Díaz-Giménes, J. (1999). Linear quadratic approximation: An introduction. Computational Methods for the Study of Dynamic Economies.

Friedman, E., Johnson, S., Kaufmann, D., and P. Zoido-Lobaton (2000). Dodging the grabbing hand: The determinants of the unnoficial activity in 69 countries. Journal of Public Economics, 76:459-493.

Gollin, D. (2002). Getting income shares right. Journal of Political Economy, 110(2):458-474.

Gomes, V., M. Bugarin, and R. Ellery Jr. (2005). Long run implications of the brazilian capital stock and income estimates. Brazilian Review of Econometrics, 25(1).

Hansen, G. \& Wright, R. (1996). The labor market in real business cycle theory. The Rational Expecations Revolution.

Judd, K. L. (1998). Numerical methods in economics. The MIT Press.

Lucas, R. E., J. (1990). Suply-side economics: An analytical review. Economic Papers, 42:293-316. 
Lucas, R. E., J. (2000). Inflation and welfare. Econometrica, 68(2):247-274.

McGrattan, E. R. \& Prescott, E. (2005). Taxes, regulations, and the value of u.s. corporations: A general equilibrium view. Review of Economic, 72:767-796.

Parente, S. L. \& Prescott, E. (2000). Barriers to riches. Cambridge, MIT Press.

Prescott, E. (2002). Prosperity and depression. American Economic Review, 92:1-15.

Schneider, F. \& Enste, D. (2000). Shadow economies: Size, cause, and consequences. Journal of Economic Literature, 38:77-114.

Slemrod, Joel, and Jon Bakija (1996). Taxing ourselves. Cambridge, MA: MIT Press.

Uhlig, H. (1999). A toolkit for analysing nonlinear dynamic stochastic models easily. Computational Methods for the Study of Dynamic Economies.

\section{A. MÉTODO NUMÉRICO}

Há vários métodos de resolver modelos de crescimento dinâ mico. Uma classe de métodos resolve os modelos com uma aproximaçã o linear perto do equilíbrio de longo prazo (estado estacionário). Ver, por exemplo, (Uhlig, 1999) e (Díaz-Giménes, 1999). Para o problema investigado neste trabalho, tais aproximações não são os melhores métodos para avaliar os efeitos quantitativos da reforma. Em geral, aproximações perto do longo prazo são úteis apenas para avaliar mudanças temporárias do equilíbrio de longo prazo, mas não para investigar uma mudança permanente de um equilíbrio para o outro. Exatamente o que acontece com uma reforma permanente.

0 método utilizado neste trabalho foi o algoritmo secante, que resolve sistemas de equações não lineares. O método consiste em calcular as derivadas parciais do Jacobiano do sistema através de uma aproximação numérica (ver (Judd, 1998)). O método resolve a transição de um equilíbrio para o outro. Nós sabemos o ponto inicial, o ponto final, e as equações que descrevem a transição. Calculam-se os efeitos assumindo que a transição tem uma duração limitada de T. No caso dos resultados acima $\mathrm{T}=50$. Exercícios de sensibilidade em relação a $\mathrm{T}$ mostram que, valores de $\mathrm{T}$ acima de 50 têm efeitos insignificantes nos resultados. 